\title{
Transition to Chaos of Buoyant-Thermocapillary Convection in Large-Scale Liquid Bridges
}

\author{
Jia Wang ${ }^{1} \cdot$ Di Wu $^{1} \cdot$ Li Duan $^{1,2} \cdot$ Qi Kang $^{1,2}$ (D)
}

Received: 8 March 2019 / Accepted: 20 November 2019 / Published online: 8 January 2020

(C) Springer Nature B.V. 2020

\begin{abstract}
To cooperate with the Chinese TG-2 space experiment project, the transition routes to chaos of buoyant-thermocapillary convection were experimentally researched in large-scale liquid bridge of $2 \mathrm{cSt}$ silicone oil with $20 \mathrm{~mm}$ in diameter. A Nanovoltmeter with high resolution was adopted to measure the dynamical temperature oscillations, as well as to detect the convective transitional behaviors which were nonlinear and non-stationary. The existence of the quasi-periodic route and the Feigenbaum route has been confirmed in large-scale LBs, and a novel periodic oscillation state was discussed in detail for the first time. The chaotic characteristics were verified by analyzing the maximum Lyapunov exponent and correlation dimension on the basis of a phasespace reconstruction. Additionally, it was found that bifurcation could potentially lead to the reconstruction of flow fields.
\end{abstract}

Keywords Thermocapillary convection $\cdot$ Large-scale liquid bridge $\cdot$ Transition route $\cdot$ Temperature oscillation $\cdot$ Chaotic dynamic analysis

\section{Introduction}

Thermocapillary convection driven by surface tension has attracted increasing attention in the fields of material science (Tang et al. 2001; Lappa 2009), chemical engineering, and space manufacturing (Kang et al. 2016; Jiang et al. 2017; Li et al. 2019). As a typical microgravity fluid system, the thermocapillary convection in liquid bridges (hereafter referred to as LB) has been researched in theory, simulation and experiment. Since 2016, a space experiment regarding the thermocapillary convection of LB has been launched on

This article belongs to the Topical Collection: Thirty Years of Microgravity Research - A Topical Collection Dedicated to J. C. Legros Guest Editor: Valentina Shevtsova

Li Duan

duanli@imech.ac.cn

Qi Kang

kq@imech.ac.cn

1 Key Laboratory of Microgravity, Institute of Mechanics, Chinese Academy of Sciences, Beijing 100190, People's Republic of China

2 School of Engineering Sciences, University of Chinese Academy of Sciences, Beijing 100049, People's Republic of China the Chinese Tiangong 2, obtaining a wealth of experimental results.

Simplified from the half-floating zone methods, LB refers to some liquid which has been limited to two coaxial rods by surface tension. Convection can be generated in the zone when there are temperature differences applied on the upper and lower rods (Ueno et al. 2003; Yano et al. 2012). Once the temperature difference exceeds a certain threshold, the fluid will oscillate periodically, which can heavily affect the quality of the growth crystals.

Based on the background of practical applications, most of the previous researches have concentrated on the critical condition of instability (Ostrach 1982; Albanese et al. 1995; Levenstam and Amberg 1995; Chen and Hu 1998), which is the initial period of the transition process to turbulence. It has been confirmed that the oscillation should be the function of many relevant parameters (Watanabe et al. 2014), such as geometric parameters (Leypoldt et al. 2000), Prandtl (Pr) numbers ( $\mathrm{Li}$ et al. 2008), and natural air convection (Wang et al. 2017; Yasnou et al. 2018). However, it has been found that, as a flow system away from the equilibrium state, when the temperature differences between the two ends of the LB exceed a certain value, dissipation structures will be built in the fields with different flow patterns. Eventually, the convection will develop into a chaotic state when the applied temperature differences become large enough. Within this flow state, 
the amplitude of the fluctuations will tend to become nonlinearly apparent in the observed features, and numerous chaotic phenomena and related nonlinear problems will emerge. These are extremely interesting issues in the field of fluid mechanics (Hu and Tang 2003). The behaviors of liquid bridges under normal gravity conditions, as well as the related routes to aperiodicity, have been previously been investigated by Chun (1984), Frank and Schwabe (1997), Ueno et al. (2003), and Melnikov et al. (2004, 2005). Similar to the term that "all roads lead to Rome", it has been found that there are also many transition routes to chaos (Mukutmoni and Yang 1995; Rahal et al. 2007; Duan et al. 2018; Yasnou et al. 2018) except for the typical double-periodic, quasi-periodic, and intermittent transition routes (Pomeau and Manneville 1980). However, the present research regarding the turbulence in LBs is far less than that of other thermocapillary convection due to the fact that the flow in LB is extremely complicated (Gollub and Benson 1980, b; Bucchignani and Mansutti 2004; Li et al. 2010; Jiang et al. 2017). As a result, up until 1995 , the routes with double-period bifurcation to chaos in LB had been confirmed in theory by $\mathrm{Hu}$ et al. It was successfully verified in small-scale LBs with diameters of 3 to $5 \mathrm{~mm}$ through ground experimentation (Yan et al. 2007; Aa et al. 2010). In 2003, Ueno et al. carefully traced the bifurcations of the hydro-thermal waves from an initial steady state to chaos. Additionally, Melnikov et al. explored quasi-periodic transition route by examining standing waves and traveling waves with mixed modes (Melnikov et al. 2004). Yasnou et al. also determined that gas flow actions could greatly affect the evolution of oscillatory states, and oscillatory regimes could potentially even vanish under the particular condition of heat transference at interfaces. However, both the space and ground experiments mentioned above were limited to smallscale LB, and the ST- 89 had confirmed that the routes to turbulent convection were complexly dependent on geometric parameters (Frank and Schwabe 1997; Schwabe and Frank 1999). Therefore, far beyond the critical conditions, via what route the thermocapillary oscillatory convection transit to turbulent or chaotic behavior in large-scale LB is still an interesting question.

Due to the limitations of space resources, the TG-2 space experiment had only observed the initial instability of the thermocapillary convection. Considering the fewer opportunities and higher costs, ground experimental research and the theoretical analyses of thermocapillary convection of largescale liquid bridges were necessary so as to provide terrestrial experimental comparisons and supplementations.

The goals of the present work is to identify various transition routes to chaos, and to analyze the chaotic state in theory. A temperature measurement system with high resolution has been designed to collect the oscillated temperature signals. Transition routes under different conditions are proven to be the Feigenbaum transition route, the quasi-periodic route and the coupled route (Wang et al. 2017), respectively. A unique periodic oscillation in the super-critical regime was analyzed in detail, which is identified in the large-scale LB for the first time. The frequencies and amplitudes for the two aforementioned routes are analyzed and compared. Additionally, the chaotic state is identified and the transition routes are quantitatively analyzed by chaotic dynamic theory.

\section{Experimental Apparatus}

For the purpose of revealing the various dynamic states in the study zone, a buoyant-thermocapillary convection system was constructed, as showed in Fig. 1.

During the experimental process, the LB of silicone oil was floated in the gap between two coaxial disks with a diameter of $20 \mathrm{~mm}$, separated by a specific distance $H$. To contend with the buoyancy convection, the temperature applied on the upper heating rod is much higher than that of the lower cooling rod. The lower cooling rod was maintained by a Peltier element. The geometrical parameters, such as the diameters $D$ of the two rods, the aspect ratio $A r=H / D$ and the volume ratio $V r=V / V_{0}$, were important for the critical point and flow structures. Where $V$ represented the liquid volume realized by the PI motor injection, and $V_{0}$ is the volume of the gap.

The temperature of the cold disk $T_{l}$ was controlled at room temperature $\left(18^{\circ} \mathrm{C}\right)$, and the temperature of the heated disk $T_{h}$ was linear heating, hence, the temperature $\Delta T$ was $\Delta T=T_{h}$ $T_{l}$. The ramping rate of the $\Delta T$ was achieved by setting the final target high temperature $T_{h}$, low temperature $T_{l}$, and heating time $t$. Here, the heating rate was $\Delta T=\left(T_{h}-T_{l}\right) /$ $t=\Delta T / t=0.3{ }^{\circ} \mathrm{C} / \mathrm{min}$, and the initial $\Delta T$ was $0{ }^{\circ} \mathrm{C}$.

Since it had been previously verified that the flow field would lose its stability when the Marangoni number exceeded approximately 20,000 (Kawamura et al. 2012; Nishino et al. 2015), 2cSt silicone oil (Shin-Etsu Chemical Co., Ltd., KF$96 \mathrm{~L}$ ) was selected as the working liquid. Its related physical properties are detailed in Table 1. In order to prevent liquid leakage, small chamferings were designed near the ends of both disks.

The influencing factors were combined into dimensionless parameters with different physical meanings for the purpose of describing the characteristics of the flow. It should be noted that the temperature dependence of the fluid viscosity cannot be neglected, especially with a large $\Delta T$. The average values of the heating and cooling temperatures were chosen as the temperature values in the empirical formulas (Formulas (1 and 2)) to obtain the viscosity of the silicone oil:

$$
\begin{aligned}
& T=\left(T_{\text {heat }}+T_{\text {cool }}\right) / 2 \\
& \frac{v(T)}{v_{0}}=\exp \left(5.892 \frac{25-T}{273.15+T}\right)
\end{aligned}
$$


Fig. 1 Schematic diagram of the experimental configuration

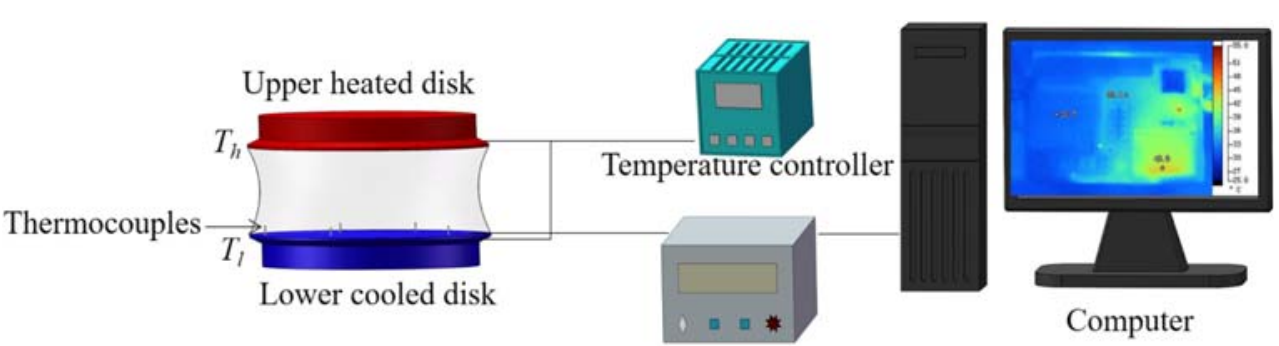

Temperature collection
Where $v_{0}$ and $T$ are the kinematic viscosity and ambient temperature at a temperature of $25^{\circ} \mathrm{C}$, respectively. To facilitate a deeper understanding of the processes, $\Delta T$ was nondimensionalized into the Marangoni number representing the influence of the surface tension, which can be defined as follows:

$M a=\frac{\left|\sigma_{T}\right| \Delta T H}{\rho \bar{v} \alpha}=\operatorname{Pr} \cdot R e$

A temperature collection system equipped with a Keithley 2812 digital multimeter and an Agilent 34970A digital multimeter was used to collect the historical temperatures. The temperature resolution of the Keithley 2812 multimeter was $0.001{ }^{\circ} \mathrm{C}$ and that of Agilent $34970 \mathrm{~A}$ was $0.01{ }^{\circ} \mathrm{C}$. The sampling rate adopted in the experiment was $10 \mathrm{~Hz}$, and thermocouples were applied to measure the temperature oscillations in the zone, located on the circumference $(15 \mathrm{~mm}$ in diameter) of the lower disk. The temperatures of the upper heated and lower cooled rods were recorded by the remaining two thermocouples and were then fed back to the control system. Managed by PID controllers (EUROTHERM 3504), the temperature differences were applied on the disks with an accuracy rate higher than $0.05^{\circ} \mathrm{C}$ achieved.

\section{Results and Discussion}

Chaos is a universal behavior of nonlinear systems. Considering its significance in theory and applications, its spatial and temporal complexity for nonlinear systems has attracted increasing attention. In this study, the flow processes from steady states to unstable states were analyzed. The quasiperiodic, double-period, and coupled transition routes were observed, and the flow characteristics of each regime in the transition process were analyzed in theory.

\section{Transition Routes to Chaos in Large-Scale LBs}

It was observed that the fluid in the study zone had accumulated more and more energy with the growing temperature differences during the transition route from a stable state to a chaotic state. The heat movement of the fluid molecules was aggravated, which had led to the drastic changes in the direction and rate of the fluid velocity. These factors had greatly affected the heat transmission. The temperature signals inner LBs had presented different oscillation forms, as shown in Fig. 2(a), which had qualitatively reflected the intensities of the flow field oscillations.

The Hilbert-Huang transform (HHT) method was chosen as the data-processing method (Huang 2005). As a superior method for time-frequency analyses of nonlinear and nonstationary data, the basis of the HHT method was found to be adaptive and the frequencies could be defined by a differentiation process. These signals could be decomposed into a series of physical quantity, and the time and frequency resolutions were no longer limited by the uncertainty principle. The time-frequency representations of the original signals were successfully obtained by combining the instantaneous frequencies and amplitude of the intrinsic mode functions.

Flow State $\mathrm{Rg} 1$ is the steady state; $\mathrm{Rg} 2$ is the periodic oscillation; $\operatorname{Rg} 3 \mathrm{a}$ represents the quasi-periodic state with two incommensurate frequencies $f_{1}$ and $f_{2}$ and their linear combinations; $\operatorname{Rg} 4$ is a quasi-periodic state with three incommensurate frequencies $f_{1}, f_{2}$ and $f_{3}$ and their linear combinations; $\operatorname{Rg} 5$ is a unique periodic oscillation; $\operatorname{Rg} 6$ represents the chaotic state.

It has been observed that, as one of the classical transition routes to chaos, the most important characteristic of quasiperiodic transition in the Landau theory (Landau 1944) is that there are an infinite number of irreducible frequencies within the dynamic system. In 1978, it was demonstrated by the Ruelle-Takens-Newhouse (RTN) Theory that a flow system can potentially directly develop into a chaotic state after three

Table 1 Physical properties of the silicone oil

\begin{tabular}{lllllll}
\hline Silicone oil (cSt) & $v\left(\mathrm{~m}^{2} \cdot \mathrm{s}^{-1}\right)$ & $\beta\left({ }^{\circ} \mathrm{C}^{-1}\right)$ & $\kappa\left(\mathrm{m}^{2} \cdot \mathrm{s}^{-1}\right)$ & $\rho\left(\mathrm{kg} \cdot \mathrm{m}^{-3}\right)$ & $\sigma\left(\mathrm{N} \cdot \mathrm{m}^{-1}\right)$ & $\partial \sigma / \partial \mathrm{T}\left(\mathrm{N} \cdot \mathrm{m}^{-1} \cdot{ }^{\circ} \mathrm{C}^{-1}\right)$ \\
\hline 2 & $2 \mathrm{e}-6$ & $1.24 \mathrm{e}-3$ & $7.00 \mathrm{e}-8$ & 873 & $1.83 \mathrm{e}-2$ & $-7.16 \mathrm{e}-5$ \\
\hline
\end{tabular}


bifurcations (Newhouse et al. 1978). In Fig. 2, an overview of the transition process with the increasing temperature difference $\Delta T$ in large-scale LB is presented for the case of $A r=$ 0.18 and $V r=0.85$. In accordance with the characteristics of the temperature oscillations, the entire process was categorized into six regimes ( $\mathrm{Rg} 1$ to 6), as detailed in Fig. 3. Once $\Delta T$ had exceeded the threshold, the flow loses its stability to periodically oscillate in the critical region, as can be seen in Fig. 3(b). Irreducible frequencies had emerged in the super critical region with the increasing $\Delta T$ (Fig. 3(c), (d), and the other frequencies could be linearly combined expressed by the fundamental frequencies (Melnikov et al. 2004). However, the quasi-periodic transition routes for large-scale LBs were observed to be quite different. For example, rather than evolving into chaos or noisy quasi-periodic routes with three frequencies (Yasnou et al. 2018), the flow had entered into a unique oscillation state (Fig. 3(e)), which was further analyzed in detail later.

Another classic transition route is the double-periodic transition route in which the flow will develop into a chaotic state after a series of periodic bifurcations. This route had been previously observed in the small-scale LB experiments (Yan et al. 2007), and was subsequently verified in theory by (Tang and $\mathrm{Hu}$ 1995) It is believed that the bifurcation points should satisfy the universal theorem. This is to say, the critical

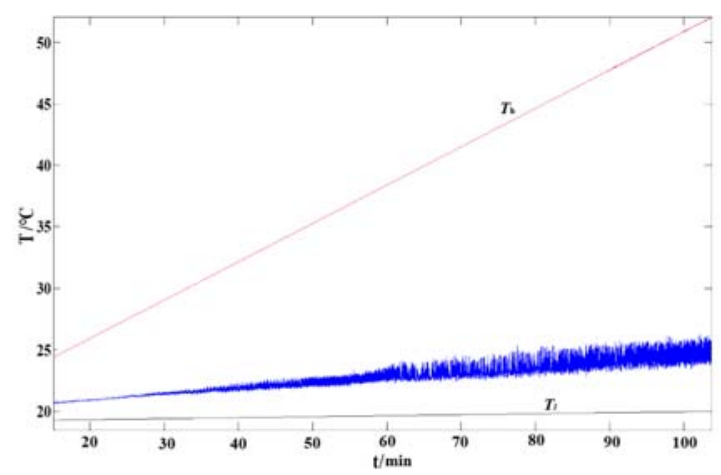

(a)

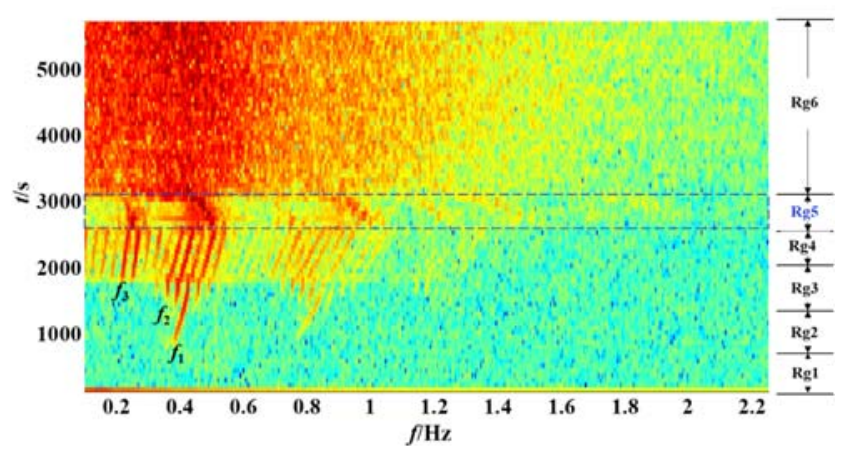

(b)

Fig. 2 Temperature oscillation signals and frequency-time analysis results: (a) Temperature oscillation signals in the quasi-periodic route (long-term trend removed); (b) Frequency-time analysis results in the quasi-periodic route. Note: $A r=0.18 ; V r=0.85$ bifurcation spacing ratio will be constant. In regard to the large-scale LBs examined in this study, the double-periodic transition route had been confirmed and analyzed its dynamic characteristics.

According to the Feigenbaum general theorem of wave spectrums in transition routes from stability to chaos, the constraint $a_{\mathrm{n}}$ should approach asymptotically:

$\delta=\lim _{n \rightarrow \infty} \frac{a_{\mathrm{n}}-a_{\mathrm{n}-1}}{a_{\mathrm{n}+1}-a_{\mathrm{n}}}=4.6692016$

For the case of $A r=0.20, V r=0.84$, the Feigenbaum constant $\delta$ can be solved by these transition processes, and the relationships can be written as follows:

$\delta_{\mathrm{n}}=\left(a_{\mathrm{n}+1}-a_{\mathrm{n}}\right) /\left(a_{\mathrm{n}+2}-a_{\mathrm{n}+1}\right) \rightarrow \delta$

Where

$$
\begin{aligned}
\delta_{1} & =\left(\Delta T_{f 2}-\Delta T_{f 1}\right) /\left(\Delta T_{f 4}-\Delta T_{f 2}\right) \\
& =(15.16-7.85) /(16.74-15.16)=4.6 \pm 0.1
\end{aligned}
$$

Which was observed to be similar to the value given by Feigenbaum Theorem.

According to the temperature oscillations in the liquid and the Fourier spectrum of the surface temperature variation, the entire process of thermocapillary convection can be divided into six regimes as follows: Steady state/periodic oscillation; $1 / 2$ double state/periodic oscillation; $1 / 4$ double state/periodic oscillation; unique periodic oscillations; and finally, a chaotic state.

It has been found that, not only in the double-periodic and quasi-periodic routes, but also in the unique transition routes of large-scale LB, coupled routes, unique periodic oscillations emerge before chaos. The coupled transition routes refer to the route co-existing with quasi-periodic and Feigenbaum bifurcation in chaos (Wang et al. 2017). The flow oscillates periodically with two fundamental frequencies $\left(f_{1}, f_{2}\right)$ in $\operatorname{Rg} 3$. Meanwhile, in the following regime, $\mathrm{n} / 2 f_{1}$ has also emerged in the field, and the regime was named $\mathrm{RgC}$. Then, after a quasi-periodic state with three incommensurate frequencies, the flow was observed to enter a unique periodic oscillation state, and eventually evolved into chaos.

\section{Introduction of a Novel Periodic Oscillation State}

The novel periodic oscillation appears in the temperature field after several bifurcations in both the double-periodic and the quasi-periodic transition route, in which the flow state had been somewhat regular.

At the beginning of the flow, the convection was observed to be in a periodic oscillation state. The phase trajectory at that time had been approximately circular, which had indicated that there is only one cycle of the system. The attractor was 
Fig. 3 A quasi-periodic transition process: (a) Time histories of the temperature oscillations; (b) Corresponding power spectra at different Marangoni numbers
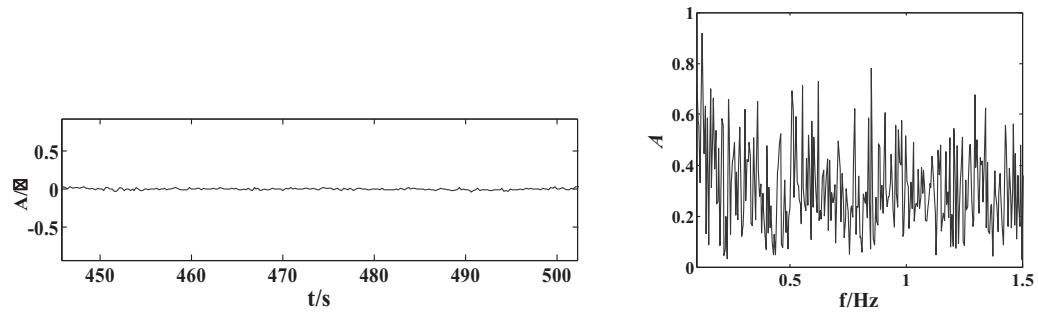

(a) $\operatorname{Rg} 1$ : steady state without oscillation, $M a=9321$
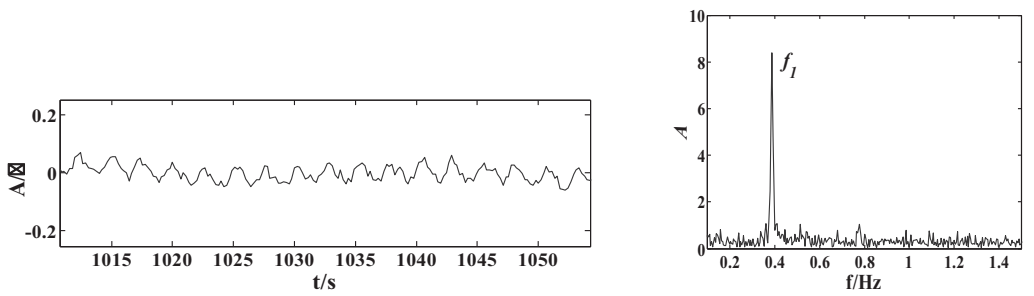

(b) Rg2: periodic oscillation, $M a=28117$
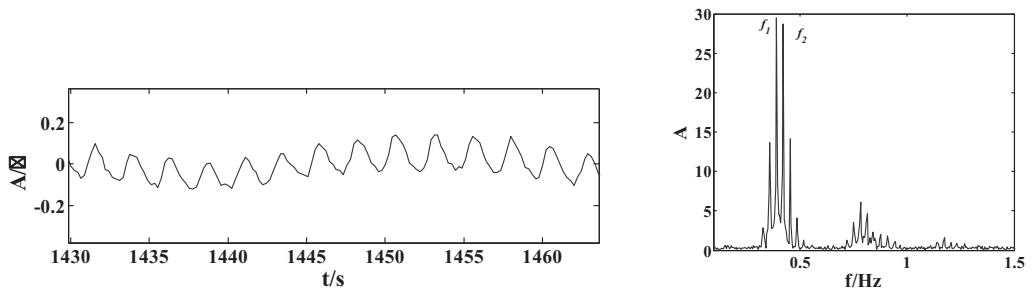

(c) Rg3: a quasi-periodic oscillation state with two incommensurate frequencies, $M a=33509$
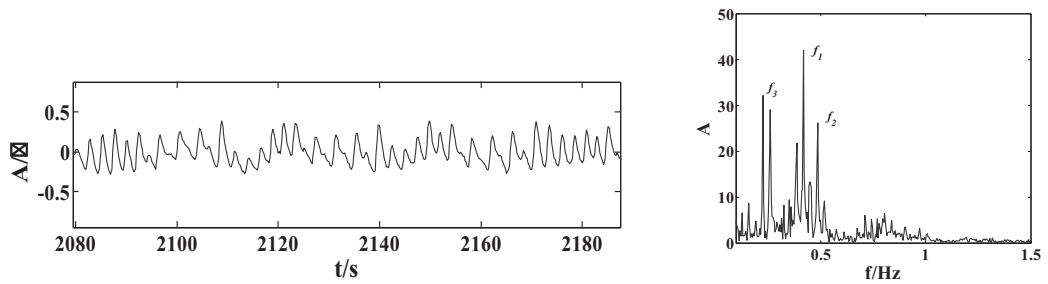

(d) Rg4: a quasi-periodic oscillation state with three incommensurate frequencies, $M a=43448$
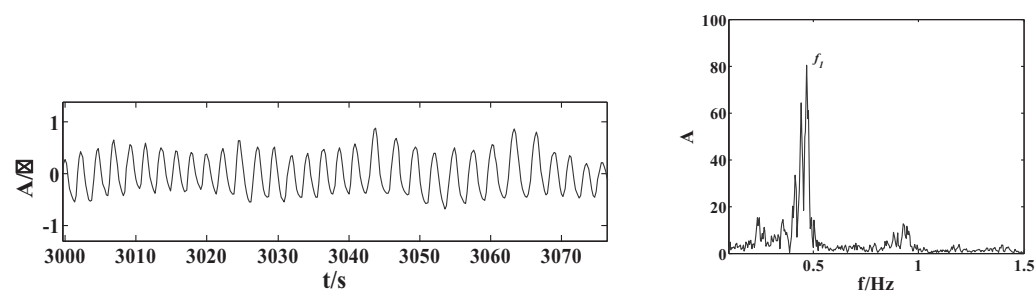

(e) $\operatorname{Rg} 5$ : a new periodic oscillation, $M a=62144$
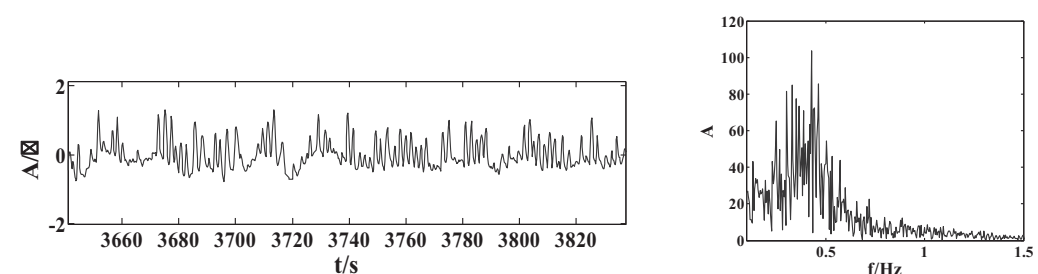

(f) Rg6: chaotic state, $M a=67453$ 
also referred to as the periodic attractor. As shown in Fig. 4(b), when $M a=34,000$, the flows had entered into a quasi-periodic regime with several fundamental frequencies state. Thereafter, as the temperature difference increased, the phase trajectory in that regime was the quasi-periodic attractor. These findings were similar to the quasi-periodic attractor obtained in the studies conducted by Dubois et al. However, when $M a=$ 62,500 , it was observed that the flows had evolved into the unique periodic motion regime, which was similar to that of (a), but the amplitude was much larger. Furthermore, when $M a=67,500$, the flows had entered into a chaotic state, and the attractor could no longer be seen in the structure. It was found that the traces were mixed, and the attractor had developed into a chaotic attractor.

Different from the periodic oscillations at the beginning of instability flow state ( $\mathrm{Rg} 2)$, the novel periodic oscillations had occurred in the supercritical regimes (Rg5) with extremely large Marangoni numbers. The thermal motion in the novel state had become faster and the amplitude had reached $0.30{ }^{\circ} \mathrm{C}$, which was much larger than the $0.01{ }^{\circ} \mathrm{C}$ of the periodic regime $(\mathrm{Rg} 2)$. The oscillation periods in that regime could be only 12 to $25 \%$ of that observed in the Rg2, as detailed in Fig. 3. There was merely one fundamental frequency found in the periodic oscillations, while many frequencies were observed in the novel state. Furthermore, it had been reflected in the temperature signals that the flows in the periodic regimes were more regular than that in the supercritical regimes $(\operatorname{Rg} 5)$. Meanwhile, the peak-to-peak values of the periodic oscillations were basically the same, while those of the $\operatorname{Rg} 5$ had displayed slight differences.

Ueno et al. (Ueno et al. 2003) observed the undeveloped chaotic flows in small-scale LB. Also, Zhu et al. (2011) obtained the non-periodic oscillations in rectangular liquid pools, and had determined that both fundamental frequencies were buried in broadband noise. However, it was observed in the present study that there were evident differences between the undeveloped chaotic flows and the novel periodic oscillations. For example, flows within the three examined routes had sinusoidally oscillated with the restricted amplitude prior to reaching the final chaotic state. Furthermore, the fundamental frequency of that regime had been further enlarged following the previous bifurcation state. Although there were many other frequencies observed, the fundamental frequency was found to be relatively higher in the spectra. Therefore, similar to the steady flows observed following the quasi-periodic oscillations in the Rayleigh-Bénard convections (Mukutmoni

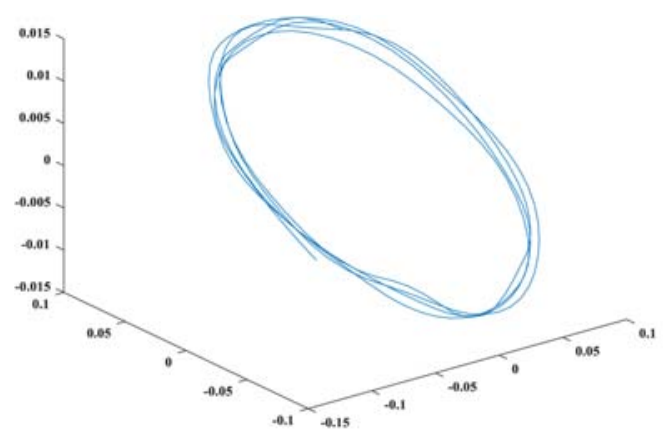

(a)

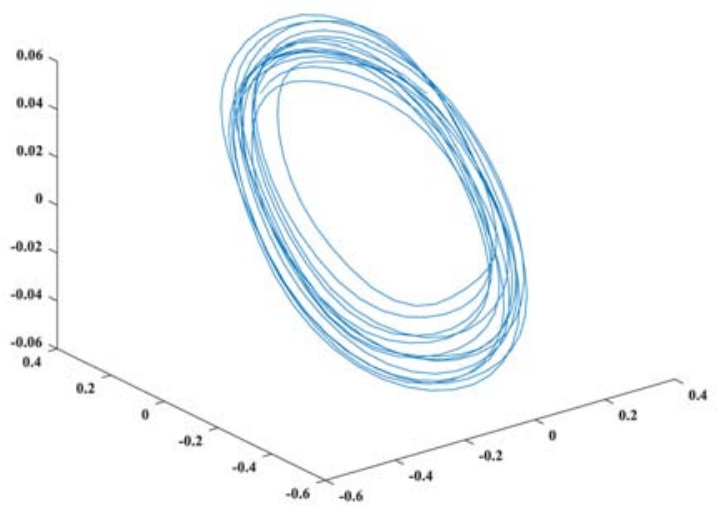

(c)

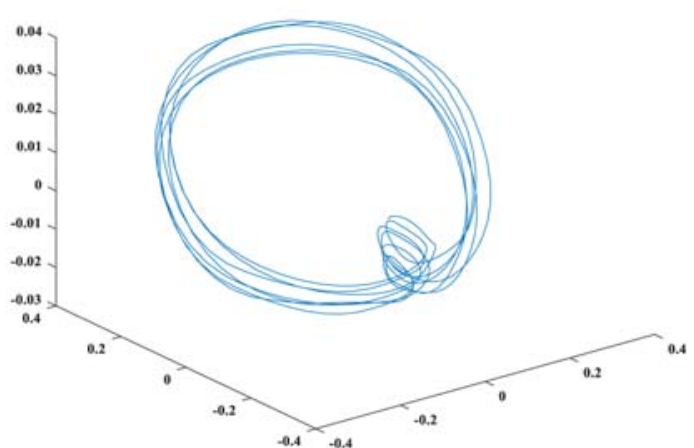

(b)

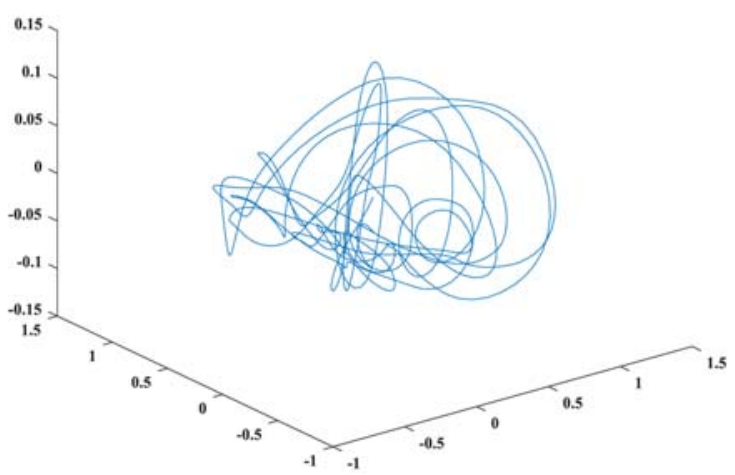

(d)

Fig. 4 Corresponding phase trajectories with the increasing Marangoni numbers during the transition to a chaotic state 
and Yang 1995), it was assumed that the emergence of the novel flow could be attributed to the variations in the average velocity fields and the spatial distributions of the temperature fields in the transition to a chaotic state. Due to the enhancement of the chaotic characteristics of the aforementioned fields, the flows in $\mathrm{Rg} 4$ failed to transition into a quasiperiodic oscillation state that with more fundamental frequencies observed in the quasi-periodic and coupled routes (or a $1 / 8$ double-periodic oscillation in the double-periodic routes). Flow in $\operatorname{Rg} 5$ is still periodic motions. The back transition from a three-frequency state or a $1 / 4$ double periodic oscillation state to a single frequency state (transition from $\mathrm{Rg} 4$ to $\mathrm{Rg} 5$ ) is similar to the phenomena of "crisis" in the case of RayleighBénard convection (Paul et al. 2011). As discussed, this kind of bifurcation can be viewed as a sudden change in the size of the attractor (Kitano et al. 1984). To further understand its characteristics, chaotic characteristics had already been confirmed by chaotic dynamic analysis results, as detailed in the following section.

Different from classic transition routes (Gollub and Benson 1980, b; Rahal et al. 2007), the founding of this phenomenon had indicated that the transition routes in largescale LB had displayed universality and particularity. The transition routes to the chaotic state were observed to be richly diversified.

\section{Results of the Chaotic Dynamic Analyses}

The amplitude and frequencies of the temperature oscillations are considered to be the characteristic parameters of dynamical states. These can be used to quantitatively represent the ranges and intensities of the oscillations and to describe the velocities of the temperature periodic motions. Figure 5 shows that the amplitude of the three routes examined had increased linearly with the increasing Marangoni number. It was found that, although the experimental conditions were different and the oscillation states had also varied, the amplitude of the temperature oscillations had remained almost consistent under certain Marangoni number. The amplitude could be expressed as a function of Marangoni number, which was independent of the geometric parameters and transition routes.

After the thermocapillary convection had been destabilized to an oscillating state, it was observed that the oscillation frequencies of the temperatures had not remained constant as the temperature differences increased. Figure 6 summarizes the changes in the oscillation frequencies with Marangoni number among these three transition routes. Although the frequencies could increase with the increasing Marangoni number, there were many differences in frequencies of the three transition routes. For example, the flow field structures of the quasi-periodic and double-periodic routes were determined to be simpler than those of the coupled routes (Wang et al. 2017). The frequency-Marangoni curves of the former two routes were found to be parallel, which had indicated that the frequencies increased linearly with the temperature differences. Meanwhile, for the coupled route, the increasing slopes with the Marangoni numbers had suggested multiple regime distribution. When $M a<27,500$, the frequency increased with a slope of $4.95 \mathrm{e}-6$ (Line: $L 1$ ). Then, the slope dramatically increased to $2.94 \mathrm{e}-6$ (Line: $L 2$ ) when $27,500<M a<31,000$, and finally decreased to $1.83 \mathrm{e}-6$ (Line: $L 3$ ), displayed an Stype distribution.

However, in supercritical regimes with large Marangoni number, the frequency distribution characteristics in that state could not be determined by the above-mentioned analysis method due to the overlapping of multiple frequencies. Therefore, further in-depth study of the frequencies was required using other analytical methods.

It has been determined that the fractal structures and characteristics of the time-dependent dynamics of a nonlinear dynamical system can be quantized using fractal numbers (Zhu et al. 2011) and the Lyapunov exponents, respectively (Zhang et al. 2014). Both can quantitatively characterize the evolution of the transition process, which could potentially change with the correlative parameters. In order to distinguish the deterministic chaos states from the random states, the fractal correlation dimension in this study was produced using a GP algorithm (Ueno et al. 2003) as follows:

$$
\begin{aligned}
C_{\mathrm{m}}(r) & =\lim _{N \rightarrow \infty} \frac{1}{N^{2}} \sum_{i, j=1, i \neq j}^{N} \theta\left(r-\left|v_{i}-v_{j}\right|\right) \\
S\left(\tau_{d}\right) & =\frac{1}{N} \sum_{i=1}^{N} \log \left(\frac{1}{M} \sum_{k_{i}=1}^{M} \frac{\left|v\left(t+\tau_{d}\right)-v\left(t+\tau_{d}\right)\right|}{\left|v(t)-v\left(k_{i}\right)\right|}\right)
\end{aligned}
$$

Where $\theta$ is the Heaviside function, and the maximum Lyapunov exponent can be evaluated by calculating the increases in the averaged divergence rates of the logarithmic distances.

The correlation dimension is defined as the slope of the straight-line segment of the double logarithmic curve $\ln C(r)$ $\ln r$. As shown in Fig. 7, as the embedding dimension $\mathrm{m}$ increased, the correlation dimension gradually increased and eventually converged to 6.27 . The convergence of the correlation dimension is an important feature which can be used to distinguish chaotic signals from random signals. Therefore, although the time-domain diagram of the oscillatory signals in Rg6 (Fig. 2) were very similar to random signals, the convergence of the correlation dimension had once again proven that the temperature oscillations observed in the experiment had eventually evolved into chaos rather than into random signals (Fig. 8, Wang et al. 2017; Fig. 9, Zhu et al. 2011).

Similarly, this study was also calculated the correlation dimension (Fig. 10a) and maximum Lyapunov exponent (Fig. 10b) of the oscillatory signals for each regime of these three transition routes. It indicated that the thermocapillary 
Fig. 5 Time histories of the temperature oscillations and corresponding power spectra in the supercritical regimes (Rg5) of the three examined routes
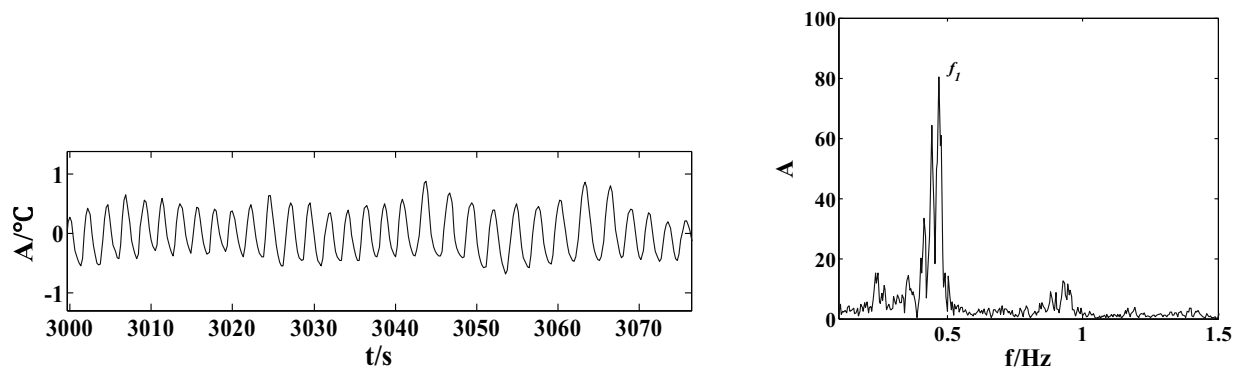

(a) $M a=62144$, quasi-periodic bifurcation transition route
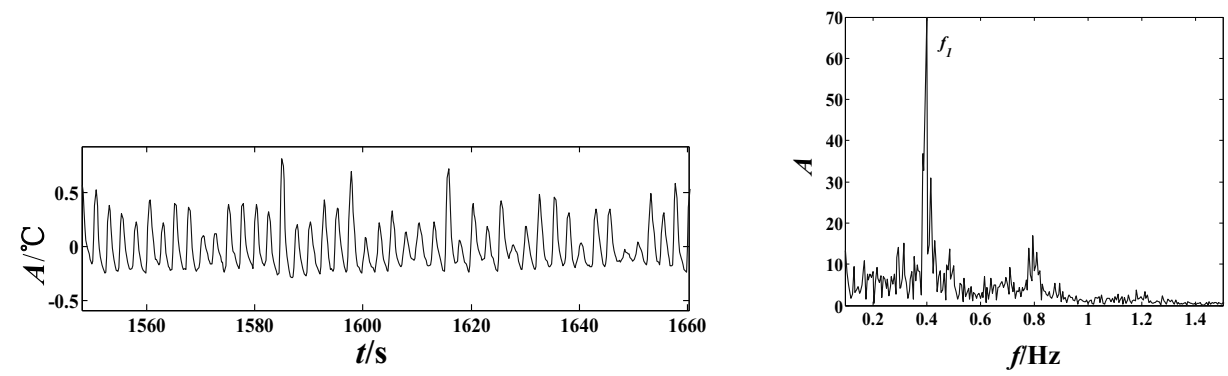

(b) $M a=45145$, double-periodic bifurcation transition route
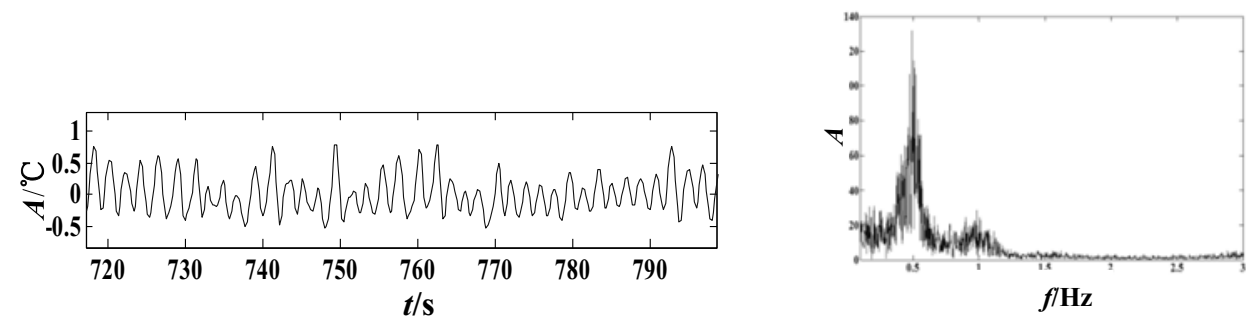

(c) $M a=61317$, the coupled transition route

flow was fractal in structure and sensitive to the initial conditions of the attractor in the pseudo-phase space. Since the

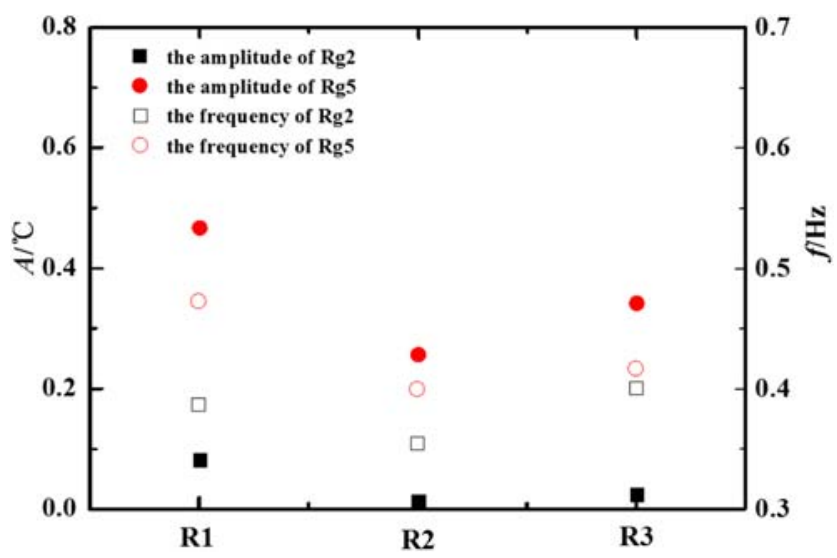

Fig. 6 Oscillatory amplitudes and frequencies of the periodic (Rg2) and supercritical $(\operatorname{Rg} 5)$ regimes in the three examined transition routes: Route 1 indicates the double-periodic route; Route 2 indicates the quasi-periodic route; Route 3 indicates the coupled route existence of the correlation dimension and the positive Lyapunov exponents were important symbols of chaos, it had been once again confirmed that the thermocapillary flows in the examined transition routes had eventually evolved into chaos.

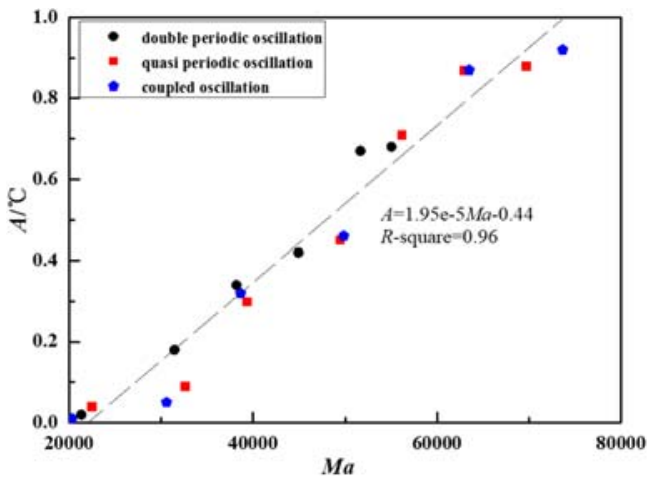

Fig. 7 Relationship between the amplitudes and Marangoni numbers in the three examined transition routes 


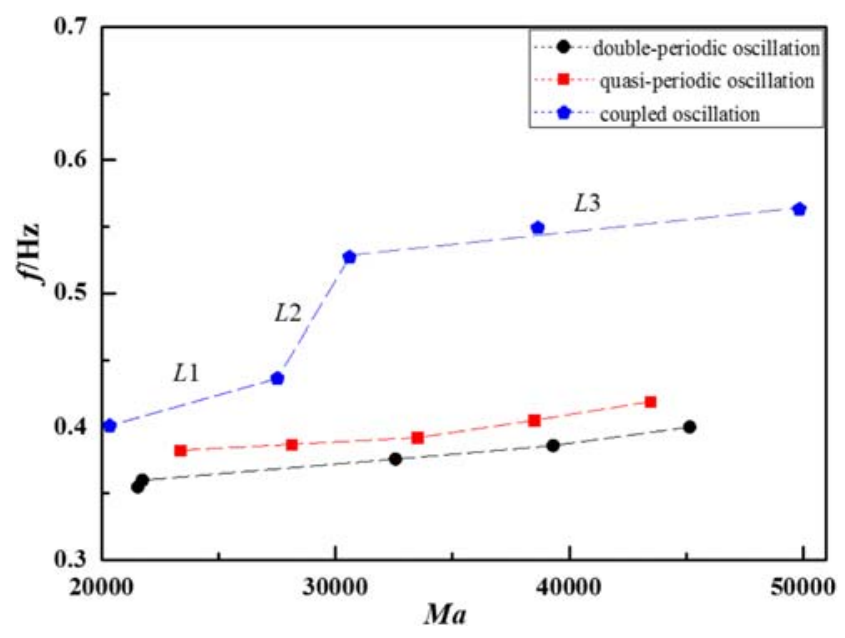

Fig. 8 Changes in the frequencies with the Marangoni numbers in the three examined transition routes (Wang et al. 2017)

As Marangoni number increased, both exponents had shown increasing tendency, indicating the chaotic characteristics of the flows become increasingly obvious. In the change diagram, a mutation zone is indicated by the dashed lines in the figure. This mutation zone corresponded to $\mathrm{Rg} 2-5$ ( $\mathrm{RgC}$ in the coupled route) during the transition process of the thermocapillary convection, which is a region with frequent bifurcations. Therefore, it can be inferred that bifurcation will lead to a sudden change in the aforementioned two exponents, indicating that the flow field has been reconstructed.

The correlation dimensions in each regimes of the three transition routes were found to be almost the same. However, the maximum Lyapunov number of the coupled transition route was found to be much lower than that of the other two routes. Furthermore, the temperature oscillations in the coupled transition routes were observed to be more regular, and the system had displayed greater stability.

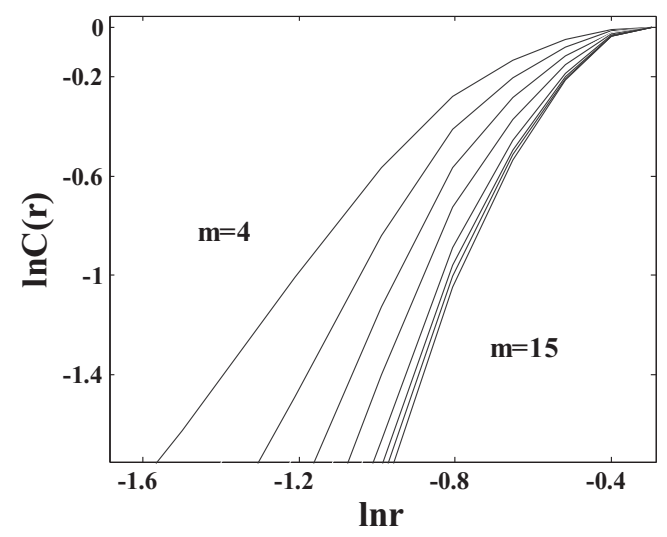

Fig. 9 Correlation dimension computation (Zhu et al. 2011)
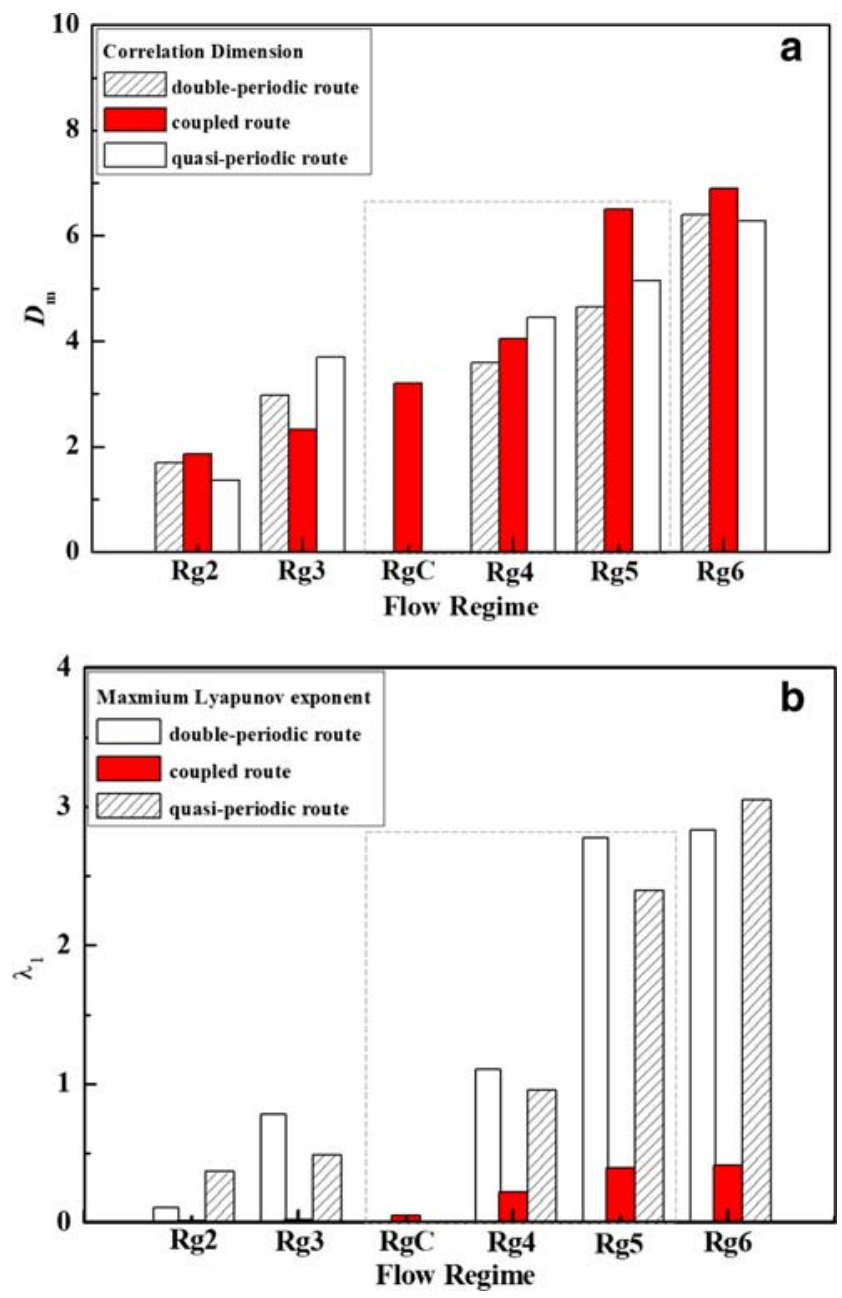

Fig. 10 a Relationships between $D_{m}$ and the different flow regimes. Note: The ideal correlation dimensions of $\operatorname{Rg} 2$ should be $D_{\mathrm{m}}=1$, and that of $\operatorname{Rg} 3$ should be $D_{\mathrm{m}}=2$. However, the non-integer values are caused by the non-ideal measurement and uncertainties of the calculation procedure of the correlation dimension (b) Relationship between $\lambda_{1}$ and the different flow regimes

\section{Conclusions}

In order to cooperate with the TG-2 space experiment, ground experimental research had conducted on the transition routes from steady states to chaotic states of thermocapillary convection in large-scale LB.

The increasing temperature differences between the cooling ends and heating ends in the experimental process have introduced the initial perturbation to the flow fields. The flow was observed to be unstable, which has resulted in periodic oscillation. The further increasing the temperature differences had expanded the external factor interference, as a result, the flow fields had entered into oscillation states with three incommensurate frequencies emerging in the fields after two hopf bifurcations. Finally, turbulent states had developed under the joint effects of the buoyancy and surface tension. Meanwhile, transition routes coupled with double-periodic 
oscillation and quasi-periodic oscillation had been first confirmed in the large-scale LB. And the transition routes in the large-scale LB were observed to be different from the typical RTN and Feigenbaum route, due to the fact that novel periodic oscillations has appeared after two transitions. These findings had confirmed the diversity of transition routes to chaos in the large-scale LB. Emergence under large Marangoni number, the fluid in this novel regime fluctuates violently. This state is found to be neutrally stable, not lasted for a long term, and would directly evolve into chaotic states with the further increasing temperature differences. The maximum Lyapunov exponents and correlation dimensions were analyzed for various dynamical behaviors. The results had indicated that the oscillatory convection had eventually entered into a chaotic state. The maximum Lyapunov exponent was positive demonstrated that the flows in the LB had been sensitive to the initial conditions. And was confirmed by the calculated correlation dimensions. Meanwhile, both had increased with the growing temperature differences. The further development of the fluid flows had enhanced the associated correlation of the dynamical system. Furthermore, it was determined that the bifurcation had caused the reconstructions of the flow fields, which had resulted in the mutations of the aforementioned two parameters.

Carrying out experiments on large-scale liquid bridges should be an extremely effective way to study the transition routes to chaos. Research on transition route to chaos has contributed to instability mechanism of the thermocapillary convection.

Acknowledges This research study was funded by the China Manned Space Engineering program (TG-2), the National Natural Science Foundation of China (U1738116, $11,372,328$ and 11,502,271), the Project funded by China Postdoctoral Science Foundation (2019 M660812), the Natural Science Foundation of Shandong Province (ZR2018BA022) and the Strategic Priority Research Program on Space Science, Chinese Academy of Sciences: SJ-10 Recoverable Scientific Experiment Satellite (XDA04020405 and XDA04020202-05).

\section{References}

Aa, Y., Li, K., Tang, Z.M., et al.: Period-doubling bifurcations of the thermocapillary convection in a floating half zone[J]. Science China Physics, Mechanics and Astronomy. 53(9), 1681-1686 (2010)

Albanese C, Carotenuto L, Castagnolo D, et al. First results from[J]. Onset" Experiment During D2 Space Mission," in Scientific Results of German Spacelab Mission D-2, PR Sahm, MH Keller, and B. Schiewe, eds., DARA, Bonn, 1995: 247-258
Bucchignani, E., Mansutti, D.: Horizontal thermal convection of succinonitrile: steady state, instablities, and transition to chaos. Physical Review E. 69(5), 056319 (2004)

Chen, Q.S., Hu, W.R.: Influence of liquid bridge volume on instability of floating half zone convection[J]. Int. J. Heat Mass Transf. 41(6-7), 825-837 (1998)

Chun C.H., Verification of turbulence developing from the oscillatory Marangoni convection in a liquid column, Proc. 5th European Symp. On Material Science and Microgravity, Schloss Elmau, 5-7 Nov. 1984, ESA SP-222: 271-280

Duan, L., Duan, L., Jiang, H., Kang, Q.: Oscillation transition routes of buoyant-Thermocapillary convection in annular liquid layers[J]. Microgravity Science and Technology. 30(6), 865-876 (2018)

Frank, S., Schwabe, D.: Temporal and spatial elements of thermocapillary convection in floating zones[J]. Exp. Fluids. 23(3), 234-251 (1997)

Gollub, J.P., Benson, S.V.: Many routes to turbulent convection. J. Fluid Mech. 100, 449-470 (1980)

Wenrui Hu, Zemei Tang, Floating Zone Convection in Crystal Growth Modeling, Science Press, Beijing, New York, 2003, pp. 8-12

Huang, N.E.: Introduction to the Hilbert-Huang transform and its related mathematical problems. Hilbert-Huang Transform Applications. 5, 1-26 (2005)

Jiang, H., Duan, L., Kang, Q.: A peculiar bifurcation transition route of thermocapillary convection in rectangular liquid layers. Exp. Thermal Fluid Sci. 88, 8-15 (2017)

Kang, Q., Duan, L., Zhang, L., Yin, Y., Yang, J., Hu, W.: Thermocapillary convection experiment facility of an open cylindrical annuli for SJ10 satellite. Microgravity Sci. Technol. 2(28), 123-132 (2016)

Kawamura, H., Nishino, K., Mastumoto, S.: Report on microgravity experiments of Maragoni convection aboard international space station. Trans. ASME J. Heat Transfer. 134, 031005-031018 (2012)

Kitano, K., Yabuzaki, T., Ogawa, T.: Symmetry-recovering crises of chaos in polarization related optical bistability. Phys. Rev. A. 29(3), 1288-1296 (1984)

Landau, L.D.: On the problem of turbulence[C]//Dokl. Akad. Nauk SSSR. 44(8), 339-349 (1944)

Lappa M. Thermal convection: patterns, evolution and stability, 2009, John Wiley

Levenstam, M., Amberg, G.: Hydrodynamic instabilities of thermocapillary flow in half zone. Fluid Mech. 297, 357-372 (1995)

Leypoldt, J., Kuhlmann, H.C., Rath, H.J.: Three-dimensional numerical simulation of thermocapillary flows in cylindrical liquid bridges[J]. J. Fluid Mech. 414, 285-314 (2000)

Li, K., Xun, B., Imaishi, N., et al.: Thermocapillary flows in liquid bridges of molten tin with small aspect ratios[J]. Int. J. Heat Fluid Flow. 29(4), 1190-1196 (2008)

Li, Y.S., Chen, Z., Zhan, J.: Double-diffusive Marangoni convection in a rectangular cavity: Transition to chaos. International Journal of Heat and Mass Transfer. 53(23/24), 5223-5231 (2010)

Li, J., Wang, H., Shichao, L.: A novel phosphorus-silicon containing epoxy resin with enhanced thermal stability, flame retardancy and mechanical properties. Polym. Degrad. Stab. 164, 36- 45 (2019)

Melnikov, D.E., Shevtsova, V.M., Legros, J.C.: Onset of temporal aperiodicity in high Prandtl number liquid bridge under terrestrial conditions. Phys. Fluids. 16(5), 1746-1757 (2004)

Melnikov, D.E., Shevtsova, V.M., Legros, J.C.: Route to aperiodicity followed by high Prandtl-number liquid bridge. 1-g case. Acta Astronautica. 56(6), 601-611 (2005)

Mukutmoni, D., Yang, K.T.: Thermal-convection in small enclosure-an atypical bifurcation sequence. International of Heat and Mass Transfer. 38(1), 113-126 (1995)

Newhouse, S., Ruelle, D., Takens, F.: Occurrence of strange AxiomA attractors near quasi periodic flows on $\mathrm{T} \mathrm{m}, \mathrm{m} \geqq 3[\mathrm{~J}]$. Commun. Math. Phys. 64(1), 35-40 (1978)

Nishino, K., Yano, T., Kawamura, H., Matsumoto, S., Ueno, I., Ermakov, M.K.: Instability of thermocapillary convection in long liquid 
bridges of high Prandtl number fluids in microgravity. J. Crystal Growth. 420, 57-63 (2015)

Ostrach, S.: Low-gravity fluid flows[J]. Annu. Rev. Fluid Mech. 14(1), 313-345 (1982)

Paul, S., Wahi, P., Verma, M.K.: Bifurcations and chaos in large-Prandtl number Rayleigh- Benard convection. Int. J. Non-Linear Mech. 46, 772-781 (2011)

Pomeau, Y., Manneville, P.: Intermittent Transition to Turbulence in Dissipative Dynamical Systems. Communications in Mathematical Physics. 74, 189-197 (1980)

Rahal, S., Cerisier, P., Abid, C.: Transition to chaos via the quasiperiodicity and characterization of attractors in confined BenardMarangoni convection. Eur. Phys. J. B. 59(4), 509-518 (2007)

Schwabe, D., Frank, S.: Experiments on the transition to chaotic thermocapillary flow in floating zones under microgravity[J]. Adv. Space Res. 24(10), 1391-1396 (1999)

Tang, Z.M., Hu, W.R.: Fractal features of oscillatory convection in the halffloating zone[J]. Int. J. Heat Mass Transf. 38(17), 3295-3303 (1995)

Tang, Z.M., Hu, W.R., Imaishi, N.: Two bifurcation transitions of the floating half zone convection. Int. J. Heat Mass Transf. 44, 1299 1307 (2001)

Ueno, I., Tanaka, S., Kawamura, H.: Oscillatory and chaotic thermocapillary convection in a half-zone liquid bridge[J]. Phys. Fluids. 15(2), 408-416 (2003)

Wang, J.,Wu, D., Duan, L., Kang, Q.: Ground experiment on the instability of buoyant-thermocapillary convection in large-scale liquid bridge with large Prandtl number. Int. J. Heat Mass Transf. 108, 2107-2119 (2017)
Wang, J., Duan, L., Kang, Q.: Oscillatory and chaotic bouyantthermocapillary convection in a large scale liquid bridge. Chin. Phys. Lett. 34(7), 074703 (2017)

Watanabe, T., Melnikov, D.E., Matsugase, T., et al.: The stability of a thermocapillary-buoyant flow in a liquid bridge with heat transfer through the interface[J]. Microgravity Science and Technology. 26(1), 17-28 (2014)

Yan, A., Zhong-Hua, C., Wen-Rui, H.: Transition to Chaos in the floating half zone convection[J]. Chin. Phys. Lett. 24(2), 475 (2007)

Yano, T., Nishino, K., Kawamura, H., Ueno, I., Matsumoto, S., Ohnishi, M.: Masato. Sakural, 3-D PTV measurement of Marangoni convection in liquid bridge in space experiment. Exp. Fluids. 53, 9-20 (2012)

Yasnou, V., Gaponenkoab, Y., Mialduna, A., Shevtsovaa, V.: Influence of a coaxial gas flow on the evolution of oscillatory states in a liquid bridge. Int. J. Heat Mass Transf. 123, 747-759 (2018)

Zhang, L., Duan, L., Kang, Q.: An experimental research on surface oscillation of buoyant-thermocapillary convection in an open cylindrical annuli. Acta Mechanica Sinica. 30(5), 681-686 (2014)

Zhu, P., Zhou, B., Duan, L., et al.: Characteristics of surface oscillation in thermocapillary convection[J]. Exp. Thermal Fluid Sci. 35(7), 1444-1450 (2011)

Publisher's Note Springer Nature remains neutral with regard to jurisdictional claims in published maps and institutional affiliations. 